\title{
Resurgence of human immunodeficiency virus and treponema pallidum co-infection
}

\begin{abstract}
The focus of this paper is to concisely review literature that investigated the resurgence of co-infection with human immunodeficiency virus and Treponema pallidum as an undeniable global epidemic in both homosexual and heterosexual communities. Issues of causation, complications, and circumvention are analyzed. Drawing from the review, the authors call for a more clinically conscious approach to mitigate this surging epidemic. Specifically, we argue that a more comprehensive and creative model is needed to respond to this rising epidemic. Proposed is the essential task for providers to carefully identify predisposing factors of this co-infection, both as primary prevention as well as for the ability to urgently, but effectively, intervene within the affected population. Concluded was that healthcare providers must not only take into consideration the epidemiology of this co-infection in affected patients, they must also utilize best treatment methodologies such as education, counseling, and repeated screening of those patients who are identified as belonging to the at-risk populations.
\end{abstract}

Keywords: HIV, Human immunodeficiency virus, Syphilis, Co-infection, Epidemic, Immuno suppression, Treponema pallidum, Sexually transmitted disease, STD
Volume 5 Issue 7 - 2017

\author{
Newton David W,' Kelly Stashauna, ${ }^{4}$ Henry \\ Suzette,' Bentley Ryan, ${ }^{3}$ Meloche Theresa \\ M,' Brown Tony L' \\ 'Major General Hugh G Robinson Center for Medicine, USA \\ ${ }^{2}$ Harvard University, USA \\ ${ }^{3}$ Western Michigan University, USA \\ ${ }^{4}$ University of Science Art and Technology, USA
}

Correspondence: Tony L Brown, Harvard University, USA, Email tbrown@post.harvard.edu

Received: August 04, 2017 | Published: November 20, 2017
Abbreviations: HIV, Human Immunodeficiency Virus; DTH, Delayed Type Hypersensitivity; IV, Intravenous; STD, Sexually Transmitted Disease; CCR5, C-C Chemokine Receptor Type 5; MSM, Men Having Sex With Men

\section{Introduction}

The noticeable spike in the prevalence of co-infection with human immunodeficiency virus (HIV) and Treponema pallidum (syphilis) has generated renewed interest in the subject. While it has been well-established that HIV infection does exert some effect on the natural history of syphilis, it was not until the recent resurgence in the contraction of syphilis by HIV patients that researchers were able to document the impact that this disease has on the course of the HIV infection. A correct assertion is that there exists a complex interaction between HIV and syphilis and that this interaction remains partly understood. New data, though, have emerged that augment our comprehension of the interaction between syphilis and HIV infection as it applies to the heterosexual population, bi-sexual partners, and men who have sex with men (MSM).

\section{Historical data}

During the previous decade, in both resource-rich and resourcepoor environments, there was a noticeable resurgence in the rates of syphilis among homosexual males (or MSM) ${ }^{1,2}$ This resurgence shines a light on syphilis, thereby targeting it as a re-emerging health concern of global significance. Reportedly, the global prevalence rate of syphilis is 36 million. ${ }^{3}$ Alarming to note is that the projected annual global rate of new syphilis infections (incidence) was 12 million per annum. ${ }^{4-6}$

In 2014, the United States' incidence of syphilis reportedly more than doubled, from 8,724 to $16,663(52.4 \%)$ new cases annually. ${ }^{7}$ The significance of the incidence rate to everyday life is clear. Approximately 1,388 persons will contract syphilis monthly with an average of 347 persons becoming infected weekly, which equates to roughly 50 new individuals infected daily just in the U.S. Statistical data from 2013 suggested that the rate of primary and secondary syphilis was highest among persons in the age ranges of 20-24 and 2529 years (16.1 and 15.6 cases per 100,000 population, respectively). ${ }^{8}$

Similar to the HIV epidemic, this resurgence of syphilis has predominantly affected homosexual men, intravenous (IV) drug users, and individuals who engaged in high-risk sex practices, to such a degree that co-infection with HIV and Treponema pallidum is now relatively common. ${ }^{9,10}$ There was a clear and noticeable shift in this epidemic, and of particular interest was that this shift occurred in areas of dense populations, with major points of commerce, and with well-established homosexual (MSM) populations. ${ }^{5,6,10}$ This particular group of persons infected with syphilis accounted for approximately $75 \%$ of all primary and secondary cases in the U.S. ${ }^{11}$

\section{Transmission}

Syphilis can be insidiously transmitted during direct sexual contact, oral or anal contact, via blood transfusions, and congenitally from a pregnant woman to her unborn fetus. ${ }^{12,13}$ A person who presents with syphilis sores, and is exposed to HIV, is two to five times more likely to contract HIV..$^{12,13}$ An HIV infected person is also at an increased risk of co-infection with syphilis. Populations identified as having the greatest risks include IV drug users, prostitutes and other sex workers as well as their partners and clients, pregnant women, and homosexual men.

Syphilis has been implicated as a facilitator to the transmission and acquisition of HIV infection, which strongly suggests interplay between these two diseases. The epidemiological profiling has identified a degree of synergy between these two sexually transmitted diseases (STD). HIV and syphilis were identified to co-facilitate transmission of one another. The case for biological plausibility was supported by these factors: HIV shedding facilitated by other STDs, mucosal barrier compromise, immunosuppression occurrence, and cellular recruitment of HIV susceptible cells.

Added to the co-facilitation of infection transmission were the behavioral risk factors. Already known was that the same risky behaviors for HIV transmission were the same as those responsible 
for the transmission of syphilis. The manifestation of syphilis is dependent upon the host's humoral immunity to T. pallidum and the equilibrium between delayed type hypersensitivity (DTH). A robust DTH response has been correlated with clearance of the infected organisms in a well-developed chancre. On the contrary, a strong humoral antibody response was linked to extended infection and progression of syphilis to a tertiary disease state. ${ }^{14}$ Co-infection with HIV created an additional layer of complexity. Since both pathogens are sexually transmitted, the presence of one may facilitate the likelihood of infection with the other. The quest to identify and meticulously outline any causative factors, therefore, is crucial to a responsible, timely, and quickly effective intervention in order to contend with this surging epidemic. ${ }^{15}$

\section{Causation}

Main causative factors implicated in the surge of HIV and syphilis co-infection were identified as both behavioral and geographical in nature. Metropolitan areas with well-established populations of homosexual males have been the most significantly affected by the identified shift in this epidemic.,3 California had an increase of greater than $700 \%$ increase in reported primary and secondary syphilis cases, between the years 1995 and 2005..$^{2-4}$ Approximately $80 \%$ of those cases involved MSM. Accepted is that there exists a well-established epidemic of HIV infection between MSM from large metropolitan areas, thus laying the foundation for a proportionately increasing incidence of concurrent syphilis and HIV infections. In California, approximately $60 \%$ of homosexual males with syphilis were also infected with HIV; estimated was that, in the major metropolitan regions, $20 \%-50 \%$ homosexual men with syphilis also had a concurrent HIV infection. ${ }^{2-4}$ Recent data identified a gender disparity in the incidence rates of syphilis. In 2013, men comprised $91 \%$ of all new cases of syphilis. Almost exclusively, these men identified themselves as either homosexual or bisexual. ${ }^{16}$ Vast racial and ethnic disparities were also reported among those people who had contracted syphilis. Found was that Black men were 5 times more likely to contract the disease than were White men; for Black women, the rate was 13 times greater than that of White women. ${ }^{16}$ One of the main reasons identified for this ethnic disparity among the Black community may be the shame factor associated with homosexuality, bisexualism, and transgender lifestyles. Black men who secretly engaged in bisexual relationships who contract this disease then spread it their female partners, predominantly Black, without their knowledge. This culture of shame and clandestineness has resulted in both males and a female in the Black community has resulted in an increased risk of co-infection with HIV and syphilis. Recently suggested was that the secrecy among homosexual and bisexual men has been a prominent factor that has contributed to the rise of the epidemic. $^{17}$

Another area of causation reportedly was the failure to disclose sexual identity and sexual habits among partners, which has been implicated in the rapid rise in the incidence of HIV and syphilis coinfections among homosexual males. Reportedly, the reasons for nondisclosure were complex. Researchers asserted that the surge was secondary to a decrease in safe sex practices. ${ }^{5}$ Current antiretroviral therapy, an increased utilization of the internet to facilitate sexual encounters among total strangers, the frequency of finding sex partners with the same HIV sero status or serosorting, and the increased use of recreational drugs -- both illicit (methamphetamines) and prescribed (Viagra) - were collectively identified as having contributed to the increased incidence of syphilis, and subsequently, the increased rates of co-infection with HIV. ${ }^{6-8}$ Lack of correct information related to the role of oral sex in the transmission of syphilis, such as having the false notion that fellatio is a safer form of sex and is seldom known to be associated with HIV transmission, also was identified as a contributing factor. ${ }^{9-11}$

\section{Complications}

When syphilis infection is in its early stages, there is the development of lesions in, on, or around the genitals. These exposed lesions are vehicles for HIV-infected bodily fluids to move unhindered in the bloodstream. ${ }^{9-11}$ Subsequently, one of the unique links between HIV and syphilis is that the expression of syphilis insults the barrier to the bloodstream provided by intact epithelium. This implies that if a syphilis-infected person has sexual relations with a person infected with HIV, then the likelihood of that person contracting HIV significantly increases.

Interestingly, this expression of syphilis highlights one of the connections between these two STDs. If left untreated this condition can lead to multiple and even irreversible complications. In people with HIV, the course of the disease may be altered when there is a syphilis co-infection. There appeared to be an increased risk of brain involvement, and complications that included ulcerations of the skin and mouth, and fever. HIV appears to enhance the course of the syphilis infection, likely due to the immunosuppressive nature of HIV that results in decreased immunological defenses against the Treponema pallidum exposure..$^{18,19}$

The immune system is pivotal in combating syphilis. ${ }^{17,20}$ Any compromise by HIV to either cell-mediated or humoral immunity, or both, could impede the host's natural defense against Treponema pallidum. ${ }^{21}$ There would be a resultant modification to the clinical expression or the natural course of syphilis infection. Recent studies have shown that syphilis has resulted in both reduced CD4 cell counts and increased viral loads in HIV-positive men. ${ }^{18,19}$ The hypothesis, then, is that similar to many other acute infections, syphilis may cause a transient increase in the viral load and a decrease in the CD4 cell count subsequent to treatment of the infection. Conceivable, therefore, is that the transitory spikes in viral load facilitated susceptibility to HIV infection among individuals who suffer from HIV and syphilis co-infection. Research on animal models revealed that there was a selective weakening of cell-mediated immunity which lead to an abbreviated incubation of syphilis, an upsurge in the number and proliferation of syphilitic lesions, and a delayed healing time. ${ }^{21}$ An explanation for the role of Treponema pallidum in the advancement of HIV transmission may suggest up-regulation of gene expression, such as that of the $\mathrm{C}-\mathrm{C}$ chemokine receptor type 5 (CCR5), used in HIV entry. ${ }^{22,23}$

When HIV-induced meningeal inflammation occurred, there was a facilitation of the penetration capability of the spirochete into the central nervous system that resulted in the development of symptomatic neurosyphilis. A review of cases that involved neurosyphilis in HIV-positive subjects in the U.S. revealed that the most frequently reported symptoms were visual disturbances (51\%); headaches $(32 \%)$; difficulty ambulating $(4 \%)$; and loss of hearing $(4 \%) .{ }^{24}$ Secondary syphilis was present in $47 \%$ of patients and $10 \%$ had signs of secondary syphilis within a week of the neurosyphilis diagnosis, $24 \%$ had early latent syphilis, and $18 \%$ had late latent syphilis. ${ }^{24}$

\section{Prevention}

The question of prevention techniques must be broached if any meaningful discussion related to resolution of this co-infection issue is to occur. To be effective, any attempt at prevention of the further 
expansion of this epidemic must be economically feasible, culturally appropriate, ethically unblemished, and therapeutically sound. Every attempt must be made to engage in widespread education that has the ability to reach the multiple at-risk populations, which includes both the homosexual and heterosexual communities. Since HIV and syphilis affect comparable patient groups and co-infection is common, then all syphilis-infected patients should receive early HIV testing. ${ }^{25}$

Conversely, all HIV-positive patients must also receive regular screens for possible syphilis infection, since it has been generally recognized that infection with Treponema pallidum may enhance the spread of HIV infection via an increased occurrence of genital ulcers. Primary and secondary prevention techniques and education must be employed. Early detection and intervention are essential for either HIV or syphilis infection, which then will enable interventions with the goal of primary prevention of co-infection with HIV and secondary prevention of further complications. Providers, clinicians, educators, and public health officials should be cognizant that syphilis can present with atypical signs (optic neuritis and uveitis, for instance) in the HIV-positive individual. There has been an increased rate of nontraditional presentations of primary syphilis and HIV-positive patients who have presented with the secondary disease. Secondary infection may be more virulent and may be accompanied by an increased rate of early neurological and ophthalmic involvement.

\section{Diagnosis}

Diagnosis is generally made with common serological testing methods. In the majority of instances, serology testing for syphilis was reliable in HIV infected patients, especially those who were not significantly immunocompromized. Despite this fact, the following scenarios have been implicated in being potentially problematic: ${ }^{26}$

A. Unusual serologic responses, such as higher-than-expected titers. ${ }^{27}$

\section{B. False negative results or delayed seroreactivity. ${ }^{28}$}

C. Biological false positive results, as a consequence of HIV infection and antigen cross-reactivity. ${ }^{29}$

Data from one study highlighted the geometric-mean RPR titer result as generally more elevated in HIV-infected than in nonHIV infected individuals, particularly during secondary syphilis. ${ }^{27}$ Inappropriate B-cell activation as a result of HIV co-infection may account for this fact. Either the prozone phenomena (non-visualization of agglutination as a result of surplus of antibodies interfering with the clumping of antigen-antibody complexes) or B-cell failure in late-stage HIV infection, may give rise to false negative results. ${ }^{28}$ Compromised responses to polysaccharide and protein antigens have been reported among HIV-infected individuals, making the serologic diagnosis of some infections, including syphilis, undependable. ${ }^{29}$ Although false positive results may occur, providers and other clinicians must be mindful to not attribute positive non-treponemal test results to HIV infection alone, even when titers are noticeably low. Doing so could lead to the under-treatment of syphilis cases in high-risk populations, as well as result in serious public health consequences.

A system of checks and balances should be in place to verify both accuracy and precision of diagnostic procedures. Recent studies have suggested that monitoring of CD4 counts and RPR titers may have significant value in determining the best candidates for examination of cerebrospinal fluid. ${ }^{30-32}$ Once syphilis has been diagnosed, providers then need to consider the possibility of neurosyphilis and decide whether a lumbar puncture is indicated.

\section{Pharmacologic treatment}

Neurosyphilis can occur at any stage of the disease in HIV coinfected patients. As a pharmacological intervention, these patients should be medicated with a penicillin-based regimen that is suitable for the treatment of neurosyphilis. In penicillin-sensitive patients, skin testing may be used to confirm the degree of sensitivity. ${ }^{26}$ Once sensitivity is established then the decision whether or not to proceed with desensitization should be made. If compliance to therapeutic regimen and close follow up are ensured, and the patient is not pregnant or refuses desensitization, the prescriber should consider doxycycline (Vibramycin) treatment. Suggested was that several treatment regimens were available for patients with an allergy to penicillin, including:

\section{Doxycycline $100 \mathrm{mg}$ orally twice daily for 14 days}

\section{Tetracycline $500 \mathrm{mg}$ orally four times daily for 14 days}

3. Ceftriaxone $1 \mathrm{~g}$ intramuscularly once daily for 8 to 10 days

\section{Azithromycin $2 \mathrm{~g}$ orally in a single dose. ${ }^{33}$}

\section{Conclusion}

Despite the various advancements made that have enhanced the clinical understanding of the interplay between HIV and Treponema pallidum infections, the therapeutic intervention techniques to be utilized for the treatment of co-infected patients has remained perplexing. The recent surge of the syphilis epidemic among HIVinfected patients revealed an old pathogen with new characteristics. The immunologic interplay between syphilis and HIV has been both subtle and complex. Clinically relevant differences in disease presentations, diagnosis, and therapeutic intervention strategies must be readily identified and utilized by those providers responsible for the health care of this population.

A more complete and innovative public health approach is needed in order for health officials and providers to respond adequately to this epidemic. With each effort at intervention, officials must also mindful to take into account the changes in the disease epidemiology of populations who are significantly at risk for co-infection with HIV and syphilis. Recommended for research are studies that focus on ascertaining the populations most likely to benefit from analysis of CSF, as well as the best treatment protocols in those patients afflicted with neurosyphilis. Clinicians are pivotal participants in syphilis control, because they must firstly identify and educate patients, counsel them in responsible and safe sex practices, and consistently screen those at increased risk for infection. Clinicians must maintain an increased level of suspicion for asymptomatic neurosyphilis in HIV-infected patients.

Providers should obtain a thorough history and, perform a careful physical examination, and should also utilize clinical testing (CD4cell count and RPR titers) to facilitate the decision to perform lumbar puncture. Healthcare providers must make the education and counseling a priority when they encounter at-risk patients, in order to minimize their risks of contracting STDs. Implementation of responsible-sex public service campaigns should include the advice to decrease the number of sexual partners, promote awareness of the health status of all partners and contacts and particularly the HIV and syphilis status, prevention of unsafe sexual practices for all STDs, and use of barrier protection methods to reduce the STD transfer risk.

\section{Acknowledgments}

None. 


\section{Conflicts of interest}

The authors have no conflicts of interest to disclose.

\section{References}

1. Centers for Disease Control and Prevention. Transmission of primary and secondary syphilis by oral sex-Chicago, Illinois, 1998-2002. MMWR Morb Mortal Wkly Rep. 2004;53(41):966-968.

2. Department of Health and Human Services CfDCaP, National Center for HIV, STD and TB Prevention, Division of STD Prevention. Sexually transmitted diseases surveillance 2004 supplement: syphilis surveillance report. Atlanta: Department of Health and Human Services CfDCaP, National Center for HIV, STD and TB Prevention, Division of STD Prevention. 2005.

3. World Health Organization. Global incidence and prevalence of selected curable sexually transmitted infections - 2008. 2012;(Geneva: WHO).1-20.

4. CDC. Improving sexually transmitted disease programs through assessment, assurance, policy development, and prevention strategies (STD AAPPS). Atlanta, GA: U.S. Department of Health and Human Services, CDC. 2014.

5. Primary and secondary syphilis among men who have sex with men-New York City, 2001. United States, 2003-2004. Centers for Disease Control and Prevention, MMWR Morb Mortal Wkly Rep. 2006;51(38):853-856.

6. Wong W, Chaw JK, Kent CK, et al. Risk factors for early syphilis among gay and bisexual men seen in an STD clinic: San Francisco, 2002-2003. Sex Transm Dis. 2005;32(7):458-463.

7. CDC, Sexually transmitted disease surveillance 2012. Atlanta, GA: US Department of Health and Human Services, CDC. 2014.

8. European Center for Prevention and Disease Control. sexually transmitted infections in Europe 1990-2010 (Stockholm: ECDC). 2012.

9. Paz-Bailey G, Meyers A, Blank S, et al. A case-control study of syphilis among men who have sex with men in New York City: association with HIV infection. Sex Transm Dis. 2004;31(10):581-587.

10. CDC Guidelines for prevention and treatment of opportunistic infections in HIV-infected adults and adolescents: recommendations from CDC, the National Institutes of Health, and the HIV Medicine Association of the Infectious Disease Society of America. MMWR. 2009;58(4):1-216.

11. Centers for Disease Control and Prevention. Reported STDs in the United States: 2012 National Data for Chlamydia, Gonorrhoea and Syphilis. CDC Fact Sheet (CDC). 2014.

12. Division of STD Prevention. National Center for HIV/AIDS, Viral Hepatitis, STD, and TB Prevention, Centers for Disease Control and Prevention. 2014.

13. Kofoed K, Gerstoft J, Mathiesen LR, et al. Syphilis and human immunodeficiency virus (HIV)-1 coinfection: influence on CD4 Tcell count. HIV-1 viral load, and treatment response. Sex Transm Dis. 2006;3(33):143-148.

14. Zinkernagel RM. Antiinfection immunity and autoimmunity. Ann NY Acad Sci. 2002;958:3-6.

15. HIV-1 viral load and treatment response. Sex Transm Dis 33: 143-148. 2006.

16. Zoufaly A, Onyoh EF, Tih PM, et al. High Prevalence of Hepatitis B and syphilis co-infection among newly diagnosed HIV patients in the northwest region of Cameroon. Trop Med Int Health. 2012;17:12.
17. Palacios R, Jiménez-Oñate F, Aguilar M, et al. Impact of syphilis infection on HIV viral load and CD4 cell counts in HIV-infected patients. J Acqir Immune Defic Syndr. 2007;44(3):356-359.

18. Division of STD Prevention. National Center for HIV/AIDS, Viral Hepatitis, STD, and TB Prevention, Centers for Disease Control and Prevention. 2014.

19. Buchacz Katea, Patel Pragnaa, Taylor Melaniec, et al. Syphilis increases HIV viral load and decreases CD4 cell counts in HIV-infected patients with new syphilis infections. AIDS. 2004;18(15):2075-2079.

20. Pavis CS, Folds JD, Baseman JB. Cell-mediated immunity during syphilis. Br J Vener Dis. 1978;54(3):144-450.

21. Lukehart SA, Baker-Zander SA, Lloyd RM, et al. Characterization of lymphocyte responsiveness in early experimental syphilis. II. Nature of cellular infiltration and Treponema pallidum distribution in testicular lesions. J Immunol. 1980;124(1):461-467.

22. Bowen DL, Lane HC, Fauci AS. Immunopathogenesis of the acquired immunodeficiency syndrome. Ann Intern Med. 1985;103(5):704-709.

23. Sellati TJ, Wilkinson DA, Sheffield JS, et al. Virulent Treponema pallidum, lipoprotein, and synthetic lipopeptides induce CCR5 on human monocytes and enhance their susceptibility to infection by human immunodeficiency virus type 1. J Infect Dis. 2000;181(1):283-293.

24. Theus SA, Harrich DA, Gaynor R, et al. Treponema pallidum, lipoproteins, and synthetic lipoprotein analogues induce human immunodeficiency virus type 1 gene expression in monocytes via NFkappaB activation. J Infect Dis. 1998;177(4):941-950.

25. Centers for Disease Control and Prevention (CDC). Symptomatic early neurosyphilis among HIV-positive men who have sex with men. Four cities, United States, January 2002 - June 2004. MMWR Recomm Rep. 2007;56(25):625-628.

26. Marra CM, Boutin P, McArthur JC, et al. A pilot study evaluating ceftriaxone and penicillin $\mathrm{G}$ as treatment agents for neurosyphilis in human immunodeficiency virus-infected individuals. Clin Infect Dis. 2000;30(3):540-544.

27. Hutchinson CM, Rompalo AM, Reichart CA, et al. Characteristics of patients with syphilis attending Baltimore STD clinics: Multiple highrisk subgroups and interactions with human immunodeficiency virus infection. Arch Intern Med. 1991;151(3):511-516.

28. Calonge N \& U.S. Preventive Services Task Force. Screening for syphilis infection: Recommendation statement. Ann Fam Med. 2004;(4)2:362-365.

29. Bowen DL, Lane HC, Fauci AS. Immunopathogenesis of the acquired immunodeficiency syndrome. Ann Intern Med. 1985;103(5):704-709.

30. Marra CM, Maxwell CL, Smith SL, et al. Cerebrospinal fluid abnormalities in patients with syphilis: Association with clinical and laboratory features. J Infect Dis. 2004;189(3):369-376.

31. Ghanem KG, Moore RD, Rompalo AM, et al. Lumbar puncture in HIVinfected patients with syphilis and no neurologic symptoms. Clin Infect Dis. 2009;48:816-821.

32. Libois A, De Wit S, Poll B, et al. HIV and syphilis: When to perform a lumbar puncture. Sex Transm Dis. 2007;34(3):141-144.

33. Calonge $\mathrm{N}$ and U.S. Preventive Services Task Force. Screening for syphilis infection: Recommendation statement. Ann Fam Med. 2004;2(4):362-365. 